Kyushu J. Math.

Vol. 60, 2006, pp. 47-62

\title{
ON THE CHARACTERISTIC NUMBERS OF THE MANIFOLDS IMMERSED IN THE MULTIPLE POINT SET OF A SELF-TRANSVERSE IMMERSION
}

\author{
Masayoshi KAMATA
}

(Received 8 March 2005)

\begin{abstract}
We study characteristic numbers of the manifold immersed in the multiple point set of a self-transverse immersion of a closed manifold in the sphere. The signature of the manifold immersed in the multiple point set is described as the characteristic number with L-classes.
\end{abstract}

\section{Introduction}

Let $f: V^{n} \longrightarrow W^{n+k}$ be a self-transverse immersion. We have the manifold $N_{d}$ that is immersed in the $d$-multiple point set in $W^{n+k}$ (cf. [2]). Herbert investigated properties of the manifold $N_{d}$ in [2] to study the multiple point set and Ronga obtained the important pull-back diagram of immersions including $f$ and the natural $d$-fold covering $p^{\prime \prime}: \widetilde{N}_{d} \longrightarrow N_{d}$ in [6]. The definition of the manifolds $N_{d}$ and $\widetilde{N}_{d}$ are given in Section 3 and here we call the manifold $N_{d}$ the $d$-multiple point manifold. This paper studies the characteristic numbers of the multiple point manifold of a self-transverse immersion of a closed smooth manifold in the sphere. Section 2 is devoted to constructing natural transformations from the cobordism cohomology to the ordinary cohomology that is used to describe the characteristic numbers of the multiple point manifold for a self-transverse immersion in the sphere. Section 3 is

2000 Mathematics Subject Classification: Primary 55N22;

Secondary 57R20, 57R42.

Keywords and Phrases: characteristic number; cobordism; self-transverse immersion. This article is an invited contribution to a special issue of the Kyushu Journal of Mathematics commemorating the sixtieth volume. 
concerned with a self-transverse immersion of an oriented manifold $V^{n}$ in the $(n+2 k)$ dimensional sphere $S^{n+2 k}$. We describe the characteristic numbers of the covering space $\widetilde{N}_{d}$ for the $d$-multiple point manifold $N_{d}$ as follows:

$$
\left\langle p_{\mathbf{t}}\left(\tau\left(\widetilde{N_{d}}\right)\right),\left[\widetilde{N}_{d}\right]\right\rangle=(-1)^{d-1}\left\langle\left\{e_{H}\left(v_{f}\right)\right\}^{d-1}\left\{p_{\mathbf{t}}\left(\tau\left(V^{n}\right)\right)\right\}^{d},\left[V^{n}\right]\right\rangle,
$$

where $p_{\mathbf{t}}(\xi)$ denotes the generalized Pontrjagin class of a vector bundle $\xi$ given in Section 2 and $e_{H}\left(v_{f}\right)$ is the Euler class in the ordinary cohomology theory. Furthermore, we discuss the unoriented case and we get the same formula as the oriented case for a self-transverse immersion $V^{n}$ in $S^{n+k}$ as follows:

$$
\left\langle w_{\mathbf{t}}\left(\tau\left(\widetilde{N_{d}}\right)\right),\left[\widetilde{N}_{d}\right]\right\rangle=\left\langle\left\{e_{H}\left(v_{f}\right)\right\}^{d-1}\left\{w_{\mathbf{t}}\left(\tau\left(V^{n}\right)\right)\right\}^{d},\left[V^{n}\right]\right\rangle
$$

By virtue of the above formulas we see that if $V^{n}$ is the boundary of an oriented manifold and $d$ is odd then the $d$-multiple point manifold $N_{d}$ is the boundary of an oriented manifold. Section 4 is concerned with the rational oriented cobordism class $\left[N_{d}\right]$ for a self-transverse immersion of an oriented manifold $V^{n}$ in $S^{n+2 k}$, which is described by the cobordism classes of the complex projective spaces. In Section 5 we generalize the formula of the Euler characteristic modulo 2 of the multiple point manifolds that is given in [1]. In Section 6 we study the signature of $N_{d}$ for a selftransverse immersion of an oriented manifold $V^{4 n}$ in $S^{4 n+2 k}$, which is described as

$$
\sigma\left(N_{d}\right)=\frac{(-1)^{d-1}}{d}\left\langle\left\{e_{H}\left(v_{f}\right)\right\}^{d-1}\left\{L\left(\tau\left(V^{4 n}\right)\right)\right\}^{d},\left[V^{4 n}\right]\right\rangle,
$$

where $L(\xi)$ denotes the $L$-class of a vector bundle $\xi$.

\section{The natural transformation from the cobordism cohomology to ordinary cohomology $H^{*}(\cdot)\left[\left[t_{1}, t_{2}, \ldots, t_{i}, \ldots\right]\right]$}

Let $\xi$ be an $n$-dimensional vector bundle over $X$. We consider the characteristic class with respect to a formal power series:

$$
f(x)=1+x t_{1}+x^{2} t_{2}+\cdots+x^{i} t_{i}+\cdots
$$

in the ring $H^{*}(X ; Q)\left[\left[t_{1}, t_{2}, \ldots, t_{i}, \ldots\right]\right]$ consisting of formal power series of $t_{i}$ with the coefficients in the rational cohomology group $H^{*}(X ; Q)$. Let $\xi$ be an $n$-dimensional real vector bundle. Here we consider formal indeterminate variables 
$\alpha_{1}, \alpha_{2}, \ldots, \alpha_{n}$ and the product of $f\left(\alpha_{i}\right)$ :

$$
\begin{aligned}
f\left(\alpha_{1}\right) f\left(\alpha_{2}\right) \cdots f\left(\alpha_{n}\right) & =\prod_{i=1}^{n}\left(1+\alpha_{k} t_{1}+\alpha_{k}^{2} t_{2}+\cdots+\alpha_{k}^{i} t_{i}+\cdots\right) \\
& =\sum s_{(\underbrace{1, \ldots, 1}_{i_{1}}, \ldots, 2, \ldots, 2, \ldots, \underbrace{\ell, \ldots, \ell)}_{i_{2}}}(\alpha) t_{1}^{i_{1}}, \ldots, t_{\ell}^{i_{\ell}} .
\end{aligned}
$$

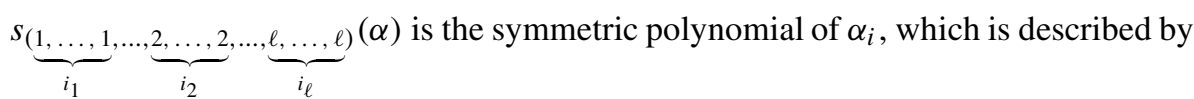
the elementary symmetric polynomials $\sigma_{i}(\alpha)$ of $\alpha_{i}$ :

$$
s_{(\underbrace{}_{i_{1}}, \ldots, 1, \ldots, 2, \ldots, 2, \ldots, \ell, \ldots, \ell)}(\alpha)=\underbrace{(1, \ldots, 1}_{i_{2}}, \ldots, \underbrace{1, \ldots, 2, \ldots, \ell_{1}^{\ell, \ldots, \ell)}}_{i_{\ell}}\left(\sigma_{1}(\alpha), \ldots, \sigma_{n}(\alpha)\right) .
$$

For the vector bundle $\xi$ we set

$$
s_{(\underbrace{}_{i_{1}}, \ldots, 1}^{1, \ldots, 2, \ldots, 2, \ldots, \ell, \ldots, \ell)}(\xi)=\underbrace{\ell}_{i_{2}}(\underbrace{1, \ldots, 1}_{i_{\ell}}, \ldots, \underbrace{2, \ldots, 2}_{i_{1}}, \ldots, \underbrace{\ell, \ldots, \ell)}_{i_{\ell}}\left(p_{1}(\xi), \ldots, p_{n}(\xi)\right) .
$$

Then the characteristic class $p_{\mathbf{t}}(\xi)$, called the generalized Pontrjagin class, can be defined as follows:

$$
p_{\mathbf{t}}(\xi)=\sum s_{(\underbrace{1, \ldots, 1}_{i_{1}}, \ldots, \underbrace{2, \ldots, 2}_{i_{2}}, \ldots, \underbrace{\ell, \ldots, \ell)}_{i_{\ell}}}(\xi) t_{1}^{i_{1}}, \ldots, t_{\ell}^{i_{\ell}} .
$$

The class $p_{\mathbf{t}}(\xi)$ satisfies the following properties.

(1) (Naturality) $p_{\mathbf{t}}\left(f^{*} \xi\right)=f^{*}\left(p_{\mathbf{t}}(\xi)\right)$, for $f: X \longrightarrow Y$.

(2) (Multiplicativity) $p_{\mathbf{t}}(\xi \oplus \eta)=p_{\mathbf{t}}(\xi) p_{\mathbf{t}}(\eta)$.

(3) For a complex line bundle $\ell$

$$
p_{\mathbf{t}}(\ell)=1+e_{H}(\ell)^{2} t_{1}+\left\{e_{H}(\ell)\right\}^{4} t_{2}+\cdots+\left\{e_{H}(\ell)\right\}^{2 n} t_{n}+\cdots
$$

where $e_{H}(\ell)$ is the Euler class of $\ell$.

The characteristic classes $s_{\omega}(\xi)$ satisfy the following properties.

(1) (Naturality) $s_{\omega}\left(f^{*} \xi\right)=f^{*}\left(s_{\omega}(\xi)\right)$, for $f: X \longrightarrow Y$.

(2) (Multiplicativity) $s_{\omega}(\xi \oplus \eta)=\sum_{\omega=\omega^{\prime} \cup \omega^{\prime \prime}} s_{\omega^{\prime}}(\xi) s_{\omega^{\prime \prime}}(\eta)$.

(3) For the canonical complex line bundle $\xi_{n}$ over the complex projective space $C P^{n}$

$$
s_{\omega}\left(\xi_{n}\right)= \begin{cases}x^{2 \ell} & \omega=(\ell) \\ 0 & \text { otherwise }\end{cases}
$$

where $H^{*}\left(C P^{n} ; Z\right)=Z\left[x_{n}\right] /\left(x_{n}^{n+1}\right)$. 
Here we see the characteristic class with respect to a formal power series:

$$
f(x)=1+x t_{1}+\cdots+x^{n} t_{n} \cdots
$$

in the ring $H^{*}\left(X ; Z_{2}\right)\left[\left[t_{1}, t_{2}, \ldots, t_{i}, \ldots\right]\right]$ consisting of formal power series of $t_{i}$ with the coefficients in the $Z_{2}$-cohomology group $H^{*}\left(X ; Z_{2}\right)$. In the same way as the generalized Pontrjagin class, we define the generalized Stiefel-Whitney class $w_{\mathbf{t}}(\xi)$. The class $w_{\mathbf{t}}(\xi)$ satisfies the following properties.

(1) (Naturality) $w_{\mathbf{t}}\left(f^{*} \xi\right)=f^{*}\left(w_{\mathbf{t}}(\xi)\right)$, for $f: X \longrightarrow Y$.

(2) (Multiplicativity) $w_{\mathbf{t}}(\xi \oplus \eta)=w_{\mathbf{t}}(\xi) w_{\mathbf{t}}(\eta)$.

(3) For a line bundle $\ell$

$$
w_{\mathbf{t}}(\ell)=1+e_{H}(\ell) t_{1}+\left\{e_{H}(\ell)\right\}^{2} t_{2}+\cdots+\left\{e_{H}(\ell)\right\}^{n} t_{n}+\cdots
$$

where $e_{H}(\ell)$ is the Euler class of $\ell$.

Now we define a natural transformation from the oriented cobordism cohomology $\Omega^{*}(X)$ to the ordinary cohomology

$$
\mathcal{P}: \Omega^{*}(X) \longrightarrow H^{*}(X ; Q)\left[\left[t_{1}, t_{2}, \ldots, t_{i}, \ldots\right]\right] .
$$

Take an element

$$
[f] \in \Omega^{n}(X)=\lim _{\vec{\ell}}\left[S^{\ell-n} X^{+}, \operatorname{MSO}(\ell)\right]_{0}, \quad f: S^{\ell-n} X^{+} \longrightarrow \operatorname{MSO}(\ell),
$$

where $\operatorname{MSO}(\ell)$ is the Thom complex of the $\ell$-dimensional universal oriented vector bundle $\gamma_{S O}^{\ell}$. The homomorphism $\mathcal{P}: \Omega^{*}(X) \longrightarrow H^{*}(X ; Q)\left[\left[t_{1}, t_{2}, \ldots, t_{i}, \ldots\right]\right]$ is defined by

$$
\mathcal{P}([f])=\sigma^{-l+n} f^{*}\left(\Phi_{\gamma_{S O}^{\ell}}\left(\frac{1}{p_{\mathbf{t}}\left(\gamma_{S O}^{\ell}\right)}\right)\right),
$$

where $\sigma^{-l+n}$ is the $(-l+n)$-times suspension isomorphism and $\Phi_{\gamma_{S O}^{\ell}}$ is the Thom isomorphism for $\gamma_{S O}^{\ell}$ in the ordinary cohomology theory. This transformation satisfies the following propositions.

Proposition 2.1. We have the following.

(1) $\mathcal{P}\left(f^{*}(a)\right)=f^{*}(\mathcal{P}(a))$, for $f: X \longrightarrow Y$.

(2) $\mathcal{P}(a b)=\mathcal{P}(a) \mathcal{P}(b)$.

(3) Let $\xi$ be an $n$-dimensional oriented vector bundle over $X$. Then

$$
\mathcal{P}(e(\xi))=e_{H}(\xi) \frac{1}{p_{\mathbf{t}}(\xi)}
$$


Proof. Here we prove part 3. In the oriented cobordism cohomology theory the Thom class $t(\xi)$ of an $n$-dimensional oriented vector bundle $\xi$ over $X$ is represented by

$$
\tilde{f}_{\xi}: T(\xi) \longrightarrow \operatorname{MSO}(n),
$$

that is, the map from the Thom complex $T(\xi)$ to $M S O(n)$ induced by the classifying map for the vector bundle $\xi$. The Euler class $e(\xi)$ in the oriented cobordism cohomology theory is represented by the composite of the zero section $s: X^{+} \longrightarrow$ $T(\xi)$ and $\tilde{f}_{\xi}$.

$$
X^{+} \stackrel{s}{\longrightarrow} T(\xi) \stackrel{\tilde{f}_{\xi}}{\longrightarrow} \operatorname{MSO}(n) .
$$

Applying $\mathcal{P}$ to the Euler class $e(\xi)$, we calculate

$$
\begin{aligned}
\mathcal{P}(e(\xi)) & =s^{*} \tilde{f}_{\xi}^{*}\left(\Phi_{\gamma_{S O}^{\ell}}\left(\frac{1}{p_{\mathbf{t}}\left(\gamma_{S O}^{n}\right)}\right)\right) \\
& =s^{*}\left(\Phi_{\xi}\left(\frac{1}{p_{\mathbf{t}}(\xi)}\right)\right) \\
& =\frac{1}{p_{\mathbf{t}}(\xi)} e_{H}(\xi) .
\end{aligned}
$$

Let $f: X \longrightarrow Y$ be a differentiable map between oriented closed manifolds. Let $h: X \longrightarrow S^{N}$ be an embedding. Then we have the normal bundle $v_{f}$ for the embedding $f \times h: X \longrightarrow Y \times D^{N}$, where $D^{N}$ is the unit disk in the Euclidean space $R^{N}$. Let $h^{*}(\cdot)$ be $\Omega^{*}(\cdot)$ or $H^{*}(\cdot)\left[\left[t_{1}, t_{2}, \ldots, t_{n}, \ldots\right]\right]$. Then $v_{f}$ is $h^{*}$ orientable. Then the Umkehrung homomorphism is the following composite:

$$
f_{!}^{h}: h^{*}(X) \stackrel{\Phi}{\longrightarrow} \tilde{h}^{*}\left(T\left(v_{f}\right)\right) \stackrel{j^{*}}{\longrightarrow} \tilde{h}^{*}\left(Y^{+} \wedge S^{N}\right) \stackrel{\sigma^{-N}}{\longrightarrow} h^{*}(Y)
$$

where $\Phi$ is the Thom homomorphism and $j$ is the natural projection as follows:

$$
j: Y^{+} \wedge S^{N}=\frac{Y \times D^{N}}{Y \times S^{n-1}} \longrightarrow \frac{Y \times D^{N}}{Y \times D^{N}-\operatorname{Int} D\left(v_{f}\right)}=T\left(v_{f}\right),
$$

where $D\left(v_{f}\right)$ denotes the disk bundle associated with the bundle $v_{f}$.

PROPOSITION 2.2. The Umkehrung homomorphisms are combined by $\mathcal{P}$ as follows:

$$
\mathcal{P}\left(f_{!}^{\Omega}(1)\right)=f_{!}^{H}\left(\frac{1}{p_{\mathbf{t}}\left(v_{f}\right)}\right)
$$

where $v_{f}$ is the virtual normal bundle for $f$. 
Proof. The element $f_{!}^{\Omega}(1)$ is represented by a map

$$
Y^{+} \wedge S^{N} \stackrel{j}{\longrightarrow} T\left(v_{f}\right) \stackrel{\tilde{f}_{v_{f}}}{\longrightarrow} \operatorname{MSO}(k)
$$

where $k=\operatorname{dim} Y+N-\operatorname{dim} X$. Then we have

$$
\begin{aligned}
\mathcal{P}\left(f_{!}^{\Omega}(1)\right) & =\sigma^{-N} j^{*} \tilde{f}_{v_{f}}^{*}\left(\Phi_{\gamma_{S O}^{k}}\left(\frac{1}{p_{\mathbf{t}}\left(\gamma_{S O}^{k}\right)}\right)\right) \\
& =\sigma^{-N} j^{*}\left(\Phi\left(\frac{1}{p_{\mathbf{t}}\left(v_{f}\right)}\right)\right) \\
& =f_{!}^{H}\left(\frac{1}{p_{\mathbf{t}}\left(v_{f}\right)}\right) .
\end{aligned}
$$

By discussing in the same way as $\mathcal{P}$, we use the generalized Stiefel-Whitney class to obtain a multiplicative natural transformation

$$
\mathcal{W}: \mathcal{N}^{*}(X) \longrightarrow H^{*}\left(X ; Z_{2}\right)\left[\left[t_{1}, t_{2}, \ldots\right]\right]
$$

where $\mathcal{N}^{*}(X)$ is the unoriented cobordism cohomology group. This transformation satisfies the following propositions.

Proposition 2.3. We have the following.

(1) $\mathcal{W}\left(f^{*}(a)\right)=f^{*}(\mathcal{W}(a))$, for $f: X \longrightarrow Y$.

(2) $\mathcal{W}(a b)=\mathcal{W}(a) \mathcal{W}(b)$.

(3) Let $\xi$ be an $n$-dimensional vector bundle over $X$. Then

$$
\mathcal{W}(e(\xi))=e_{H}(\xi) \frac{1}{w_{\mathbf{t}}(\xi)} .
$$

Proposition 2.4. Let $f: X \longrightarrow Y$ be a differentiable map between closed manifolds. Then the Umkehrung homomorphisms $f_{!}^{\mathcal{N}}$ and $f_{!}^{H_{2}}$ are combined by $\mathcal{W}$ as follows:

$$
\mathcal{W}\left(f_{!}^{\mathcal{N}}(1)\right)=f_{!}^{H_{2}}\left(\frac{1}{w_{\mathbf{t}}\left(v_{f}\right)}\right)
$$

where $v_{f}$ is the virtual normal bundle for $f$ and $f_{!}^{H_{2}}$ denotes the Umkehrung homomorphism in the ordinary cohomology with the coefficient ring $Z_{2}$.

\section{The characteristic numbers of the multiple point manifold of a self-transverse immersion}

Let $f: V^{n} \longrightarrow W^{n+k}$ be an immersion of an $n$-dimensional smooth manifold $V^{n}$ in an $(n+k)$-dimensional smooth manifold $W^{n+k}$. If $f^{-1}(y)$ consists of distinct 
$d$-points, then $y$ is said to be $d$-multiple point. Let $d f: \tau\left(V^{n}\right) \longrightarrow \tau\left(W^{n+k}\right)$ be the tangent map for $f$ that is a bundle homomorphism between the tangent bundles $\tau\left(V^{n}\right)$ and $\tau\left(W^{n+k}\right)$. For any multiple point $y \in f\left(V^{n}\right)$ with $f^{-1}(y)=\left\{x_{1}, x_{2}, \ldots, x_{d}\right\}$ the images under the tangent map $d f$ of the tangent spaces $T_{x_{i}}\left(V^{n}\right)$ at $x_{i}$ are said to be in general position in the tangent space $T_{y}\left(W^{n+k}\right)$ if the intersection of $d f T_{x_{i}}\left(V^{n}\right)$ and the normal spaces $N_{x_{i}}\left(V^{n}\right)$ of $d f T_{x_{i}}\left(V^{n}\right)$ span the tangent space $T_{y}\left(W^{n+k}\right)$, that is,

$$
\bigcap_{i=1}^{d} d f T_{x_{i}}\left(V^{n}\right) \oplus\left(\bigoplus_{i=1}^{d} N_{x_{i}}\left(V^{n}\right)\right) \cong T_{y}\left(W^{n+k}\right) .
$$

If for any multiple point $y$ of an immersion $f: V^{n} \longrightarrow W^{n+k}$ the images under the tangent map of the tangent spaces at the points in $f^{-1}(y)$ are in general position in $T_{y}\left(W^{n+k}\right)$, then $f$ is said to be a self-transverse immersion. An immersion is approximated by a self-transverse immersion. Suppose that $f: V^{n} \longrightarrow W^{n+k}$ be a self-transverse immersion and $n+k-d k \geq 0$. Let

$$
M_{d}=\left\{\left(x_{1}, \ldots, x_{d}\right) \mid f\left(x_{1}\right)=\cdots=f\left(x_{d}\right), x_{i} \neq x_{j}(i \neq j)\right\} .
$$

Then $M_{d}$ is an $(n+k-d k)$-dimensional submanifold in $V^{n} \times V^{n} \times \cdots \times V^{n}$. Let the symmetric group $S_{d}$ of degree $d$ act on $M_{d}$ by

$$
\sigma\left(x_{1}, \ldots, x_{d}\right)=\left(x_{\sigma(1)}, \ldots, x_{\sigma(d)}\right), \quad \sigma \in S_{d}
$$

The orbit space is denoted by $N_{d}=M_{d} / S_{d}$, that is said to be a $d$-multiple point manifold. Let $S_{d-1}$ act on $M_{d}$ by

$$
\sigma\left(x_{1}, \ldots, x_{d}\right)=\left(x_{1}, x_{\sigma(2)}, \ldots, x_{\sigma(d)}\right), \quad \sigma \in S_{d-1},
$$

and denote the orbit space by $\widetilde{N}_{d}=M_{d} / S_{d-1}$. Then we have a $d$-fold covering $p_{d}^{\prime \prime}: \widetilde{N}_{d} \longrightarrow N_{d}$. First we recall Ronga's result [6] that plays an important role in this paper. The following pull-back diagram of immersions is said to be a sub-Cartesian:

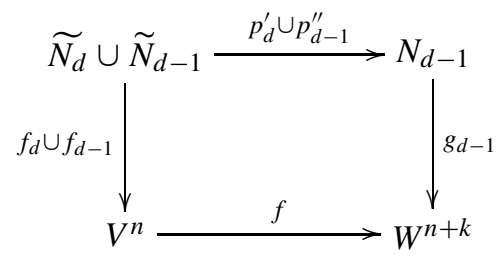


where

$$
\begin{gathered}
p_{d}^{\prime}\left(\left[x_{1}, \ldots, x_{d}\right]\right)=\left[x_{2}, \ldots, x_{d}\right] \\
p_{d-1}^{\prime \prime}\left(\left[x_{1}, \ldots, x_{d-1}\right]\right)=\left[x_{1}, \ldots, x_{d-1}\right] \\
g_{d-1}\left(\left[x_{1}, \ldots, x_{d-1}\right]\right)=f\left(x_{1}\right) \\
f_{d}\left(\left[x_{1}, \ldots, x_{d}\right]\right)=x_{1} .
\end{gathered}
$$

This diagram is a generalized example of the clean intersection diagram of the Quillen theorem [5]. Ronga obtained in [6] the following theorem.

THEOREM 3.1. (Ronga [6]) Let $h^{*}$ be a generalized cohomology theory. If each normal bundle in the above sub-Cartesian pull-back diagram of immersions is $h^{*}$-orientable, then it follows that

$$
f^{*} g_{(d-1) !}(1)=f_{d !}(1)+e\left(v_{f}\right) f_{d-1 !}(1),
$$

where $e\left(v_{f}\right)$ is the Euler class of the normal bundle for $f$.

We recall that if $f: V^{n} \longrightarrow W^{n+k}$ is a self-transverse immersion of an oriented manifold $V^{n}$ in an oriented manifold $W^{n+k}$ and $k$ is even, then $\widetilde{N_{d}}, N_{d}$ and the normal bundle are orientable (cf. [2]). As $\Omega^{i}$ (\{a point $\left.\}\right)=0$ for $i>0$, we use Theorem 3.1 to prove the following corollary.

COROLlary 3.2. If $f: V^{n} \longrightarrow S^{n+2 k}$ is a self-transverse immersion of an oriented manifold $V^{n}$ in the $(n+k)$-sphere $S^{n+2 k}$, then it follows that

$$
f_{d !}^{\Omega}(1)=(-1)^{d-1}\left\{e\left(v_{f}\right)\right\}^{d-1} .
$$

We use Corollary 3.2 to obtain the relation between Pontrjagin numbers of $\widetilde{N_{d}}$ and $V^{n}$ for a self-transverse immersion of an oriented manifold in the sphere $f: V^{n} \longrightarrow S^{n+2 k}$.

THEOREM 3.3. Let $f: V^{n} \longrightarrow S^{n+2 k}$ be a self-transverse immersion of an oriented manifold $V^{n}$. Then,

$$
\left\langle p_{\mathbf{t}}\left(\tau\left(\widetilde{N}_{d}\right)\right),\left[\widetilde{N}_{d}\right]\right\rangle=(-1)^{d-1}\left\langle\left\{e_{H}\left(v_{f}\right)\right\}^{d-1}\left\{p_{\mathbf{t}}\left(\tau\left(V^{n}\right)\right)\right\}^{d},\left[V^{n}\right]\right\rangle
$$

where $v_{f}$ is the normal bundle of $f$.

Proof. Apply $\mathcal{P}$ to the equation $f_{d !}^{\Omega}(1)=(-1)^{d-1} e\left(v_{f}\right)^{d-1}$ in Corollary 3.2, and we use Proposition 2.1(3) and Proposition 2.2 to obtain

$$
f_{d !}^{H}\left(\frac{1}{p_{\mathbf{t}}\left(v_{f_{d}}\right)}\right)=(-1)^{d-1}\left(e_{H}\left(v_{f}\right) \frac{1}{p_{\mathbf{t}}\left(v_{f}\right)}\right)^{d-1} .
$$


Note that $\tau\left(\widetilde{N}_{d}\right) \oplus v_{f_{d}} \cong f_{d}^{*}\left(\tau\left(V^{n}\right)\right)$, and $\tau\left(V^{n}\right) \oplus v_{f}$ is the trivial vector bundle. Then we have

$$
f_{d !}^{H}\left(p_{\mathbf{t}}\left(\tau\left(\widetilde{N}_{d}\right)\right)\right)=(-1)^{d-1}\left\{e_{H}\left(v_{f}\right)\right\}^{d-1}\left\{p_{\mathbf{t}}\left(\tau\left(V^{n}\right)\right)\right\}^{d} .
$$

We note that the Umkehrung homomorphism $g_{!}^{H}$ for a map $g: M \longrightarrow N$ between orientable manifolds $M$ and $N$ coincides with the following composite:

$$
H^{*}(M) \stackrel{D}{\longrightarrow} H_{*}(M) \stackrel{g_{*}}{\longrightarrow} H_{*}(N) \stackrel{D^{-1}}{\longrightarrow} H^{*}(N),
$$

where $D$ denotes the Poincare isomorphism. Evaluate both sides of $(*)$ on the fundamental class $\left[V^{n}\right]$, and we then complete the proof.

Applying the natural transformation $\mathcal{W}$ to Ronga's formula of Corollary 3.2 for the unoriented cobordism cohomology, we have

$$
f_{!}^{\mathcal{N}}(1)=\left\{e\left(v_{f}\right)\right\}^{d-1}
$$

for a self-transverse immersion $f: V^{n} \longrightarrow S^{n+k}$. Then we have the following.

THEOREM 3.4. If $f: V^{n} \longrightarrow S^{n+k}$ is a self-transverse immersion, then

$$
\left\langle w_{\mathbf{t}}\left(\tau\left(\widetilde{N}_{d}\right)\right),\left[\widetilde{N}_{d}\right]\right\rangle=\left\langle\left\{e\left(v_{f}\right)\right\}^{d-1}\left\{w_{\mathbf{t}}\left(\tau\left(V^{n}\right)\right)\right\}^{d},\left[V^{n}\right]\right\rangle .
$$

COROLLARY 3.5. If $f: V^{n} \longrightarrow S^{n+k}$ is a self-transverse immersion, then the Euler characteristic modulo 2 of the covering space $\widetilde{N}_{d}$ for the $d$-multiple point manifold is described as

$$
\chi_{2}\left(\widetilde{N}_{d}\right)=\left\langle\left\{e\left(v_{f}\right)\right\}^{d-1}\left\{w\left(\tau\left(V^{n}\right)\right)\right\}^{d},\left[V^{n}\right]\right\rangle .
$$

Proof. Let $t_{1}=1$ and $t_{i}=0$ for $i>1$ in the formula of Theorem 3.4. Then we see that the left-hand side is the Euler characteristic modulo 2 of $\widetilde{N_{d}}$.

This corollary implies the following result obtained in [4].

COROLlary 3.6. Suppose that $f: V^{n} \longrightarrow S^{n+k}$ is a self-transverse immersion. If $V^{n}$ is the boundary of a compact manifold and $d$ is odd, then the Euler characteristic of the d-multiple point manifold is even.

THEOREM 3.7. Suppose that $f: V^{n} \longrightarrow S^{n+2 k}$ is a self-transverse immersion of an oriented closed manifold $V^{n}$ in the sphere $S^{n+2 k}$. If $V^{n}$ is a boundary of an oriented compact manifold, then $\widetilde{N}_{d}$ is a boundary of an oriented compact manifold and, furthermore, if $d$ is odd, then $N_{d}$ is a boundary of an oriented compact manifold. 
Proof. Theorems 3.3 and 3.4 imply that if $V^{n}$ is a boundary of a compact manifold, then all of the Pontrjagin numbers and the Stiefel-Whitney numbers of $\widetilde{N}_{d}$ are zero. Therefore, $\widetilde{N}_{d}$ is a boundary of an oriented compact manifold [8]. By making use of the $d$-fold covering $p^{\prime \prime}: \widetilde{N}_{d} \longrightarrow N_{d}$, we have

$$
\left\langle p_{\mathbf{t}}\left(\tau\left(\widetilde{N_{d}}\right)\right),\left[\widetilde{N_{d}}\right]\right\rangle=d\left\langle p_{\mathbf{t}}\left(\tau\left(N_{d}\right)\right),\left[N_{d}\right]\right\rangle
$$

and if $d$ is odd, then

$$
\left\langle w_{\mathbf{t}}\left(\tau\left(\widetilde{N_{d}}\right)\right),\left[\widetilde{N}_{d}\right]\right\rangle=d\left\langle w_{\mathbf{t}}\left(\tau\left(N_{d}\right)\right),\left[N_{d}\right]\right\rangle
$$

and so if $d$ is odd, then all Pontrjagin numbers and Stiefel-Whitney numbers of $N_{d}$ are zero. Therefore, $N_{d}$ is a boundary of an oriented compact manifold [8].

\section{On the cobordism classes of the multiple point manifolds}

Here we consider the symmetric polynomial of the indeterminate variables $\alpha_{i}$

$$
\begin{aligned}
& s_{i_{1}}^{(1, \ldots, 1}, \ldots, \underbrace{2, \ldots, 2}_{i_{2}}, \ldots, \underbrace{\ell, \ldots, \ell)}_{i_{\ell}}(\alpha) \\
& \quad=\Sigma \alpha_{1} \alpha_{2} \cdots \alpha_{i_{1}} \alpha_{i_{1}+1}^{2} \cdots \alpha_{i_{1}+i_{2}}^{2} \cdots \alpha_{i_{1}+\cdots+i_{\ell-1}+1}^{\ell} \cdots \alpha_{i_{1}+\cdots+i_{\ell}}^{\ell} .
\end{aligned}
$$

We describe $s_{\omega}(\alpha)$ as the polynomial of elementary symmetric polynomials $\sigma_{j}$ with respect to $\alpha_{i}$ that is denoted by $s_{\omega}\left(\sigma_{1}, \sigma_{2}, \ldots\right)$. For a vector bundle $\xi$, let

$$
s_{\omega}(\xi)=s_{\omega}\left(p_{1}(\xi), p_{2}(\xi), \ldots\right),
$$

where $p_{i}(\xi)$ is the $i$ th Pontrjagin class of $\xi$. Let $C P^{n}$ be the complex projective $n$-space.

We calculate the characteristic class $s_{\omega}\left(\tau\left(C P^{n}\right)\right)$ for the tangent bundle over the projective space $C P^{n}$. The tangent bundle $\tau\left(C P^{n}\right) \oplus 1$ is isomorphic to the Whitney sum $(n+1) \xi_{n}$ of $n+1$ canonical line bundles $\xi_{n}$. We then have

$s_{(\underbrace{1, \ldots, 1}_{i_{1}}, \ldots, 2, \ldots, 2, \ldots, \ell, \ldots, \ell)}\left(\tau\left(C P^{n}\right)\right)=\frac{(n+1) !}{i_{1} ! \cdots i_{\ell} !\left(n+1-i_{1}-\cdots-i_{\ell}\right) !} x_{n}^{2\left(i_{1}+2 i_{2}+\cdots \ell i_{\ell}\right)}$

and

$$
s_{(k)}\left(\tau\left(C P^{n}\right)\right)=(n+1) x_{n}^{2 k}
$$

where $H^{*}\left(C P^{n} ; Z\right) \cong Z\left[x_{n}\right] /\left(x_{n}^{n+1}\right)$. As $\Omega_{*} \otimes Q \cong Q\left[\left[C P^{2}\right],\left[C P^{4}\right], \ldots,\left[C P^{2 n}\right]\right.$, ...], the cobordism class of $N_{d}$ is described as

$$
\left[N_{d}\right]=\sum_{i_{1}+\cdots+i_{\ell}=2 n-(d-1) k} a_{i_{1} \cdots i_{\ell}}\left[C P^{2 i_{1}}\right] \cdots\left[C P^{2 i_{\ell}}\right] .
$$


We now calculate the coefficient of the indecomposable class in the right-hand side.

THEOREM 4.1. Suppose that $f: V^{4 n} \longrightarrow S^{4 n+2 k}$ is a self-transverse immersion of an oriented closed manifold $V^{n}$ in the sphere $S^{n+2 k}$. If $(d-1) k$ is even, then

$$
\begin{aligned}
{\left[N_{d}\right]=} & \frac{(-1)^{d-1}}{2 n-(d-1) k+1}\left\langle\left\{e_{H}\left(v_{f}\right)\right\}^{d-1} s_{((2 n-(d-1) k) / 2)}\left(\tau\left(V^{4 n}\right)\right),\left[V^{4 n}\right]\right\rangle \\
& \times\left[C P^{2 n-(d-1) k}\right]+\text { decomposable elements in } \Omega_{*} \otimes Q
\end{aligned}
$$

where $e_{H}\left(v_{f}\right)$ is the Euler class of the normal bundle of $f$ in the ordinary cohomology and $\left[V^{4 n}\right]$ is the fundamental class of $V^{n}$.

Proof. We observe that the coefficient of $t_{(2 n-(d-1) k) / 2}$ of the equation given in Theorem 3.3 has

$$
\begin{aligned}
& \left\langle s_{((2 n-(d-1) k) / 2)}\left(\tau\left(\widetilde{N}_{d}\right)\right),\left[\widetilde{N}_{d}\right]\right\rangle \\
& \quad=(-1)^{d-1} d\left\langle e_{H}\left(v_{f}\right)^{d-1} s_{((2 n-(d-1) k) / 2)}\left(\tau\left(V^{4 n}\right)\right),\left[V^{4 n}\right]\right\rangle .
\end{aligned}
$$

As

$$
\left\langle s_{(2 n-(d-1) k) / 2}\left(\tau\left(C P^{2 n-(d-1) k}\right)\right),\left[C P^{2 n-(d-1) k}\right]\right\rangle=2 n-(d-1) k+1
$$

and

$$
\left\langle s_{((2 n-(d-1) k) / 2)}\left(\tau\left(\widetilde{N}_{d}\right)\right),\left[\widetilde{N}_{d}\right]\right\rangle=d\left\langle s_{((2 n-(d-1) k) / 2)}\left(\tau\left(N_{d}\right)\right),\left[N_{d}\right]\right\rangle,
$$

it follows that

$$
\begin{aligned}
& a_{2 n-(d-1) k} \\
& \quad=(-1)^{d-1} \frac{1}{2 n-(d-1) k+1}\left\langle e_{H}\left(v_{f}\right)^{d-1} s_{((2 n-(d-1) k) / 2)}\left(\tau\left(V^{4 n}\right)\right),\left[V^{4 n}\right]\right\rangle .
\end{aligned}
$$

This completes the proof.

By using the method in the proof of Theorem 4.1, we obtain the following results (cf. Szúcs [7]).

PROPOSITION 4.2. In the rational oriented cobordism ring $\Omega \otimes Q$, there are the following relations.

(1) If $f: V^{4 n} \longrightarrow S^{8 n-4}$ is a self-transverse immersion, then

$$
\left[N_{2}\right]=-\frac{1}{3}\left\langle e\left(v_{f}\right) p_{1}\left(\tau\left(V^{4 n}\right)\right),\left[V^{4 n}\right]\right\rangle\left[C P^{2}\right] .
$$


(2) If $f: V^{4 n} \longrightarrow S^{8 n-8}$ is a self-transverse immersion, then

$$
\begin{gathered}
{\left[N_{2}\right]=a\left[C P^{4}\right]+b\left[C P^{2}\right]^{2}} \\
a=-\frac{1}{5}\left\langle e\left(v_{f}\right)\left\{\left\{p_{1}\left(\tau\left(V^{4 n}\right)\right)\right\}^{2}-2 p_{2}\left(\tau\left(V^{4 n}\right)\right)\right\},\left[V^{4 n}\right]\right\rangle \\
b=\frac{1}{18}\left\langle e\left(v_{f}\right)\left\{3\left\{p_{1}\left(\tau\left(V^{4 n}\right)\right)\right\}^{2}-10 p_{2}\left(\tau\left(V^{4 n}\right)\right)\right\},\left[V^{4 n}\right]\right\rangle .
\end{gathered}
$$

\section{A remark on the Euler numbers of multiple point manifolds of a self-transverse immersion}

Let $f: V^{n} \longrightarrow S^{n+k}$ be a self-transverse immersion of a closed $n$-dimensional manifold $V^{n}$ in the $(n+k)$-dimensional sphere. In this section we generalize the result about the total sum of the Euler characteristic modulo 2 of the $d$-multiple point manifolds $N_{d}$ that is obtained by Akhmetiev et al in [1]. Let $v_{f}$ and $w_{i}\left(v_{f}\right)$ be the normal bundle of $f$ and the $i$ th Stiefel-Whitney class, respectively. The Euler characteristic modulo 2 of the covering space $\widetilde{N_{d}}$ for the $d$-multiple point manifold $N_{d}$ is described as follows.

THEOREM 5.1. We have

$$
\sum_{d \geq 1} \chi_{2}\left(\widetilde{N}_{d}\right) \equiv\left\langle\frac{1}{1+w_{1}\left(v_{f}\right)+w_{2}\left(v_{f}\right)+\cdots+w_{k-1}\left(v_{f}\right)},\left[V^{n}\right]\right\rangle .
$$

Proof. By making use of Corollary 3.5 we calculate

$$
\begin{aligned}
\sum_{d \geq 1} \chi_{2}\left(\widetilde{N}_{d}\right) & =\sum_{d \geq 1}\left\langle\left\{e\left(v_{f}\right)\right\}^{d-1}\left\{w\left(\tau\left(V^{n}\right)\right)\right\}^{d},\left[V^{n}\right]\right\rangle \\
& =\left\langle\frac{w\left(\tau\left(V^{n}\right)\right)}{1+e\left(v_{f}\right) w\left(\tau\left(V^{n}\right)\right)},\left[V^{n}\right]\right\rangle \\
& =\left\langle\frac{1}{w\left(v_{f}\right)+e\left(v_{f}\right)},\left[V^{n}\right]\right\rangle \\
& =\left\langle\frac{1}{1+w_{1}\left(v_{f}\right)+w_{2}\left(v_{f}\right)+\cdots w_{k-1}\left(v_{f}\right)},\left[V^{n}\right]\right\rangle .
\end{aligned}
$$

We use Theorem 5.1 to describe the sum of the Euler characteristics modulo 2 of the $d$-multiple point manifold $N_{d}$ for a self-transverse immersion $f: V^{n} \longrightarrow S^{n+k}$ as follows. 
COROLlary 5.2. If $n+k$ is odd, then

$$
\sum_{d \geq 1} \chi_{2}\left(N_{d}\right) \equiv\left\langle\frac{1}{1+w_{1}\left(v_{f}\right)+w_{2}\left(v_{f}\right)+\cdots+w_{k-1}\left(v_{f}\right)},\left[V^{n}\right]\right\rangle .
$$

Proof. If $n+k$ is odd and $d$ is even, then $\operatorname{dim} \widetilde{N_{d}}=\operatorname{dim} N_{d}=n-(d-1) k$ is odd and

$$
\chi_{2}\left(\widetilde{N_{d}}\right) \equiv \chi_{2}\left(N_{d}\right) \equiv 0 \quad \bmod 2 .
$$

By making use of a $d$-fold covering $p_{d}^{\prime \prime}: \widetilde{N}_{d} \longrightarrow N_{d}$, if $d$ is odd then $\chi_{2}\left(\widetilde{N}_{d}\right) \equiv$ $\chi_{2}\left(N_{d}\right)$. Therefore, Theorem 5.1 implies this corollary.

The following result is obtained immediately from Corollary 5.2.

COROLlary 5.3. Let $f: V^{n} \longrightarrow S^{n+k}$ be a self-transverse immersion of a closed $n$-dimensional manifold $V^{n}$ in the sphere $S^{n+k}$. Suppose that $n+k$ is odd and

$$
w_{1}\left(v_{f}\right)=w_{2}\left(v_{f}\right)=\cdots=w_{k-1}\left(v_{f}\right)=0 .
$$

Then we have

$$
\sum_{d \geq 1} \chi\left(N_{d}\right) \equiv 0 \quad \bmod 2
$$

\section{On the signature of multiple point manifolds}

Let $M$ be an orientable closed $4 n$-dimensional manifold and let $\left\{\alpha_{i}\right\}$ be the basis of $H^{2 n}(M ; Q)$. Denote by $\sigma(M)$ the signature of $M$ defined by the number of positive eigenvalues minus the number of negative eigenvalues for the symmetric matrix $\left[\left\langle\alpha_{i} \cup \alpha_{j},[M]\right\rangle\right]$. Let $\left\{L_{k}\right\}$ be the multiplicative sequence of polynomials belonging to the power series

$$
\frac{\sqrt{x}}{\tanh \sqrt{x}}=1+\frac{1}{3} x+\cdots+(-1)^{k-1} 2^{2 k} \frac{B_{k}}{(2 k) !} x^{k}+\cdots,
$$

where $B_{k}$ denotes the Bernoulli number. Let us consider the power series

$$
L(p(\xi))=\sum L_{k}\left(p_{1}(\xi), \ldots, p_{k}(\xi)\right) .
$$

The signature $\sigma(M)$ is described as follows [3]:

$$
\sigma(M)=\langle L(p(\tau(M))),[M]\rangle .
$$


In the same way as the construction of $\mathcal{P}$ we have a multiplicative natural transformation

$$
\mathcal{L}: \Omega^{*}(X) \longrightarrow H^{*}(X ; Q)
$$

that is defined for $f: S^{\ell-n} X^{+} \longrightarrow M S O(\ell)$ by

$$
\mathcal{L}([f])=\sigma^{-l+n} f^{*}\left(\Phi_{\gamma_{S O}^{\ell}}\left(\frac{1}{L\left(\gamma_{S O}^{\ell}\right)}\right)\right),
$$

where $\sigma$ is the suspension isomorphism and $\Phi_{\gamma_{S O}^{\ell}}$ is the Thom isomorphism with respect to $\gamma_{S O}^{\ell}$ in the ordinary cohomology theory. Then we have the following proposition.

Proposition 6.1. The natural transformation $\mathcal{L}$ satisfies the following properties.

(1) $\mathcal{L}\left(f^{*}(a)\right)=f^{*}(\mathcal{L}(a))$, for $f: X \longrightarrow Y$.

(2) $\mathcal{L}(a b)=\mathcal{L}(a) \mathcal{L}(b)$.

(3) $\mathcal{L}(e(\xi))=e_{H}(\xi)(1 / L(\xi))$.

(4) Let $f: X \longrightarrow Y$ be a differentiable map between orientable manifolds. Then

$$
\mathcal{L}\left(f_{!}(1)\right)=f_{!}^{H}\left(\frac{1}{L\left(v_{f}\right)}\right) .
$$

Applying the natural transformation $\mathcal{L}$ to the formula in Corollary 3.2, the signature of $N_{d}$ is described as follows.

THEOREM 6.2. Suppose that $f: V^{4 n} \longrightarrow S^{4 n+2 k}$ is a self-transverse immersion of an oriented closed manifold $V^{4 n}$ in the $(4 n+2 k)$-dimensional sphere $S^{4 n+2 k}$. If $(d-1) k$ is even, then

$$
\sigma\left(N_{d}\right)=\frac{(-1)^{d-1}}{d}\left\langle\left\{e_{H}\left(v_{f}\right)\right\}^{d-1}\left\{L\left(\tau\left(V^{4 n}\right)\right)\right\}^{d},\left[V^{4 n}\right]\right\rangle .
$$

Proof. Apply $\mathcal{L}$ to the formula given in Corollary 3.2 and use Proposition 6.1 to obtain

$$
\left\langle L\left(\tau\left(\widetilde{N}_{d}\right)\right),\left[\widetilde{N}_{d}\right]\right\rangle=(-1)^{d-1}\left\langle\left\{e_{H}\left(v_{f}\right)\right\}^{d-1}\left\{L\left(\tau\left(V^{4 n}\right)\right)\right\}^{d},\left[V^{4 n}\right]\right\rangle .
$$

By making use of the $d$-fold covering $p^{\prime \prime}: \widetilde{N_{d}} \longrightarrow N_{d}$ we have

$$
\left\langle L\left(\tau\left(\widetilde{N_{d}}\right)\right),\left[\widetilde{N_{d}}\right]\right\rangle=d\left\langle L\left(\tau\left(N_{d}\right)\right),\left[N_{d}\right]\right\rangle .
$$

Thus, we complete the proof. 
Let $f: C P^{2 n} \longrightarrow S^{8 n-4 k}$ be a self-transverse immersion. We calculate the signature of the double point manifold of the self-transverse immersion. By Theorem 6.2

$$
\sigma\left(\tilde{N}_{2}\right)=-\left\langle e\left(v_{f}\right) L\left(\tau\left(C P^{2 n}\right)\right)^{2},\left[C P^{2 n}\right]\right\rangle .
$$

On the other hand, we have

$$
\begin{gathered}
e\left(v_{f}\right)=\left(\begin{array}{c}
4 n-2 k \\
2 n-2 k
\end{array}\right) x_{2 n}^{2 n-2 k} \\
\left\{L\left(\tau\left(C P^{2 n}\right)\right)\right\}^{2}=\left(\frac{x_{2 n}}{\tanh x_{2 n}}\right)^{4 n+2} .
\end{gathered}
$$

$\sigma\left(\tilde{N}_{2}\right)$ is the coefficient of $x_{2 n}^{2 n}$ in the polynomial

$$
\left(\begin{array}{l}
4 n-2 k \\
2 n-2 k
\end{array}\right)\left(\frac{x_{2 n}}{\tanh x_{2 n}}\right)^{4 n+2} x_{2 n}^{2 n-2 k} .
$$

Now we calculate the coefficient of $x_{2 n}^{2 k}$ in the polynomial

$$
\left(\frac{x_{2 n}}{\tanh x_{2 n}}\right)^{4 n+2}
$$

The coefficient is given by the following integration around the origin:

$$
\frac{1}{2 \pi i} \oint \frac{1}{z^{2 k+1}}\left(\frac{z}{\tanh z}\right)^{4 n+2} d z .
$$

The substitution $u=\tanh z$, with

$$
\begin{aligned}
d z & =\frac{d u}{1-u^{2}} \\
z & =\frac{1}{2}(\log (1+u)-\log (1-u)) \\
& =u\left(1+\frac{u^{2}}{3}+\frac{u^{4}}{5}+\cdots+\frac{u^{2 k}}{2 k+1}+\cdots\right),
\end{aligned}
$$

shows that

$$
\begin{aligned}
& \frac{1}{2 \pi i} \oint \frac{1}{z^{2 k+1}}\left(\frac{z}{\tanh z}\right)^{4 n+2} d z \\
&=\sum_{\ell=0}^{k}\left\{\sum_{i_{1}+2 i_{2}+\cdots+\ell i_{\ell}=\ell} \frac{(4 n-2 k+1) !}{i_{1} ! i_{2} ! \cdots i_{\ell} !\left(4 n-2 k+1-\left(i_{1}+i_{2}+\cdots+i_{\ell}\right)\right) !}\right. \\
&\left.\quad \times\left(\frac{1}{3}\right)^{i_{1}}\left(\frac{1}{5}\right)^{i_{2}} \cdots\left(\frac{1}{2 \ell+1}\right)^{i_{\ell}}\right\}
\end{aligned}
$$


Let $d(4 n, 8 n-4 k)$ denote this number. Then we have the following result.

THEOREM 6.3. If $f: C P^{2 n} \longrightarrow S^{8 n-4 k}$ is a self-transverse immersion, then

$$
\sigma\left(N_{2}\right)=-\frac{1}{2} d(4 n, 8 n-4 k)\left(\begin{array}{c}
4 n-2 k \\
2 n-2 k
\end{array}\right) .
$$

Example. If $f: C P^{2 n} \longrightarrow S^{8 n-4}$ is a self-transverse immersion, then

$$
\sigma\left(N_{2}\right)=-\frac{2 n+1}{3}\left(\begin{array}{l}
4 n-2 \\
2 n-2
\end{array}\right) \text {. }
$$

\section{REFERENCES}

[1] P. M. Akhmetiev, R. Rimányi and A. Szúcs. A generalization of Banchoff's triple point theorem. Proc. Amer. Math. Soc. 126 (1998), 913-915.

[2] R. J. Herbert. Multiple point of immersed manifolds. Mem. Amer. Math. Soc. 34 (1981).

[3] F. Hirzeburch. Topological Methods in Algebraic Geometry, 3rd edn. Springer, Berlin, 1966.

[4] M. Kamata. On multiple points of a self-transverse immersion. Kyushu J. Math. 50(2) (1996), 275283.

[5] D. Quillen. Elementary proofs of some results of cobordism theory using Steenrod operations. Adv. Math. 7 (1971), 29-56.

[6] F. Ronga. Multiple points of smooth immersions. Comment. Math. Helv. 55 (1980), 521-527.

[7] A. Szúcs. A double points formula on complex $K$-theory and an application. Bull. London Math. Soc. 25 (1993), 184-188.

[8] C. T. C. Wall. Determination of the cobordism ring. Ann. of Math. (2) 72 (1960), 292-311.

Masayoshi Kamata

Graduate School of Mathematics

Kyushu University

Hakozaki, Fukuoka 812-8581

Japan 